\title{
A CLASS OF LINEAR TRANSFORMATIONS
}

\author{
R. V. CHACON
}

1. The purpose of this note is to construct a class of positive and invertible isometries of $L_{1}(0,1)$ which give a counterexample in ergodic theory. Specifically, we construct a class such that for each

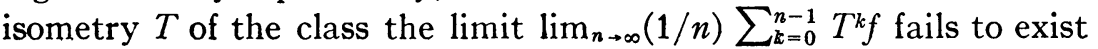
almost everywhere, for some $f \in L_{1}$. The proof is divided into two lemmas. The first gives that $\lim _{\inf } f_{n \rightarrow \infty}(1 / n) \sum_{k=0}^{n-1} T^{k} f=0$ a.e. The second lemma gives $\lim \sup _{n \rightarrow \infty}(1 / n) \sum_{k=0}^{n-1} T^{k} f=+\infty$ a.e. The second lemma also gives an example of a measurable point transformation having no $\sigma$-finite equivalent invariant measure. This example is considerably simpler than that of [5]. The proof can be modified so that if $\delta>0$ the transformations $T$ of $L_{1}(0,1)$ which are constructed satisfy also the condition that $\|T\|_{\infty} \leqq 1+\delta$.

The author wishes to thank S. Kakutani and A. and C. Ionescu Tulcea for bringing this problem to his attention.

2. We give first some definitions and introduce our notation.

Definition 1. Let $A$ and $B$ be measurable sets of the real line of positive and finite measure. The regular map $R$ of $L_{1}(A)$ onto $L_{1}(B)$ is the linear extension of the map

$$
R \psi_{A_{x}}=\frac{m(A)}{m(B)} \psi_{B_{x}},
$$

where

$$
\begin{gathered}
A_{x}=(-\infty, x) \cap A, \quad B_{x^{\prime}}=\left(-\infty, x^{\prime}\right) \cap B, \\
\frac{m\left(B_{x^{\prime}}\right)}{m(B)}=\frac{m\left(A_{x}\right)}{m(A)}
\end{gathered}
$$

and where $\psi_{A_{x}}, \psi_{B_{x^{\prime}}}$ are the characteristic functions of $A_{x}$ and $B_{x^{\prime}}$, respectively.

It follows that $R$ is a positive and invertible isometry of $L_{1}(A)$ onto $L_{1}(B)$ and that there is an invertible point transformation $\tau$ defined almost everywhere mapping $B$ "linearly" onto $A$ such that $R$ is the adjoint of the transformation from $L_{\infty}(B)$ onto $L_{\infty}(A)$ induced by $\tau$.

Received by the editors April 18, 1963. 
Definition 2. A partition of the unit interval is a finite collection $\left\{J_{k}, k=1, \cdots, N\right\}$ of pairwise disjoint (except for sets of measure zero) sets whose union is the unit interval.

Definition 3. Let $\left\{J_{k}, k=1, \cdots, N\right\}$ be a partition of the unit interval. The regular map $T$ associated with the partition is the linear operator which is the direct sum of the regular maps of $L_{1}\left(J_{k}\right)$ onto $L_{1}\left(J_{k+1}\right), \quad k=1, \cdots, N-2$, so that $T$ maps $L_{1}\left(\bigcup_{k=1}^{N-2} J_{k}\right)$ onto $L_{1}\left(\bigcup_{k=2}^{N-1} J_{k}\right)$.

Note that $T$ is a positive and invertible isometry.

3. The construction used in the proof has points of contact with the Kakutani "skyscraper" construction as well as with the methods used in [3] and [5]. The proof is based on the following two lemmas. In fact, as remarked in the introduction, Lemma 2 yields an example of the sort given in [5]. To see this, let $\tau$ be the point transformation associated with $T$ and let $r(x)$ be the Radon-Nikodym derivative of an invariant measure equivalent to Lebesgue measure. The invariance of the measure implies that we may take $p_{k} \equiv r, k \geqq 0$ in the theorem of [2] to obtain that $\lim _{n \rightarrow \infty}(1 / n) \sum_{k=0}^{n-1} T^{k} f$ exists a.e. contradicting the fact that the limit superior is infinite.

Lemma 1. Let $\left\{J_{k}, k=1, \cdots, N\right\}$ be a partition of the unit interval and let $T$ be its associated regular map. If $f \in L_{1}\left(\cup_{\boldsymbol{k}=2}^{N-1} J_{k}\right), \epsilon>0$ and $K>0$, then there exists a partition $\left\{\tilde{J}_{k}, k=1, \cdots, M\right\}$ such that its associated regular map $\tilde{T}$ is an extension of $T$, and an $n>K$ such that

$$
\left|\frac{f+\tilde{T} f+\cdots+\tilde{T}^{n-1} f}{n}\right| \leqq \epsilon
$$

on a set of measure greater than $(1-\epsilon)$.

Proof. We define $\left\{\tilde{J}_{k}\right\}$ by setting $\tilde{J}_{k}=J_{k}, k=1, \cdots, N-1$, and $\tilde{J}_{k}, k=N, \cdots, M$ so that

(i) $\tilde{J}_{k}, k=N, \cdots, M$ are pairwise disjoint and $J_{N}=\bigcup_{k=1}^{M} \tilde{J}_{k}$,

(ii) $m\left(\bigcup_{k=1}^{N} \widetilde{J}_{k}\right) \geqq(1-\epsilon / 2)$.

It follows that $\left\{\tilde{J}_{k}, k=1, \cdots, M\right\}$ is a partition of the unit interval and that its associated regular map $\tilde{T}$ is an extension of $T$. Further, for $M-N \geqq N-2+j$,

$$
\sum_{k=0}^{N-1+j} \tilde{T}^{k} f=\sum_{k=0}^{N-1} \tilde{T}^{k} f
$$

almost everywhere on $A=\bigcup_{k=1}^{N} \tilde{J}_{k}$. Since $\|\tilde{T}\| \leqq 1$ it follows that $\psi_{A} \cdot \sum_{k=0}^{N-1} \tilde{T}^{k} f \in L_{1}(A)$, and thus there exists a constant $H$ with the property that 


$$
\left|\sum_{k=0}^{N-1} \tilde{T}^{k} f\right| \leqq H
$$

almost everywhere on a subset $B$ of $A$ of measure $(1-\epsilon)$. If $M-N$ $\geqq N^{\top}-2+\max (K, H / \epsilon)$ then it follows from (1.1) that

$$
\left|\frac{1}{n} \sum_{k=0}^{n-1} \tilde{T}^{k} f\right| \leqq \frac{H}{n} \leqq \epsilon
$$

almost everywhere on $B$ for $n=[\max (K, H / \epsilon)]+N$.

LemMa 2. Let $\left\{J_{k}, k=1, \cdots, N\right\}$ be a partition of the unit interval, and let $T$ be its associated regular map. Let $\epsilon>0, K>0$, and $B>0$ be positive constants and $f$ a non-negative function in $L_{1}\left(\cup_{k=1}^{N-1} J_{k}\right)$. Then there exist integers $m_{1} \geqq K, m_{2} \geqq K$ and a partition $\left\{\tilde{J}_{k}, k=1, \cdots, M\right\}$ such that its associated regular map is an extension of $T$ and such that

$$
\sup _{m_{1} \leqq n \leqq m_{2}} \frac{\tilde{T}^{n-1} f}{n} \geqq B
$$

on a set of measure greater than $(1-\epsilon)$.

Proof. We first prove that we may assume without loss of generality

(i) $f=\delta \psi_{J_{k 0}}$, for $\delta>0$ and $1 \leqq k_{0} \leqq N-1-K$,

(ii) $m\left(\bigcup_{k=1}^{N-1} J_{k}\right) \geqq 1-\epsilon / 2$,

(iii) $m\left(J_{N-1}\right)<\max _{1 \leq k \leq N-2} m\left(J_{k}\right)$.

Since $f \in L_{1}\left(\cup_{k=1}^{N-1} J_{k}\right)$ there exists a set $A_{k_{0}} \subset J_{k_{0}}, 1 \leqq k_{0} \leqq N-1$ such that $f \geqq \delta \psi_{A_{k_{0}}}$, for $\delta>0$. Wethen form the partition $\left\{J_{k}^{\prime}, k=1, \cdots, 2 N\right\}$ obtained by setting

$$
\begin{aligned}
J_{k_{0}}^{\prime} & =A_{k_{0}}, \\
T^{-j} A_{k_{0}} & =J_{k_{0}-j}^{\prime}, \quad j=1, \cdots, k_{0}-1, \\
T^{j} A_{k_{0}} & =J_{k_{0}+j}^{\prime}, \quad j=1, \cdots, N-1-k_{0}, \\
J_{N}^{\prime} & =J_{1}-J_{1}^{\prime}, \\
J_{N+j}^{\prime} & =T^{j} J_{N}^{\prime}, \quad j=1, \cdots, N-2,
\end{aligned}
$$

and letting $J_{2 N-1}^{\prime}$ and $J_{2 N}^{\prime}$ be two disjoint sets such that $J_{2 N-1}^{\prime}+J_{2 N}^{\prime}$ $=J_{N}$ and such that $m\left(J_{2 N}^{\prime}\right) \leqq \epsilon / 2$. The associated regular map $T^{\prime}$ is clearly an extension of $T$. Since $T^{\prime}$ (and $T$ ) are positive we may take $f=\delta \psi_{A_{k 0}}$.

We suppose in what follows that $f=\delta \psi_{J_{k 0}}$ for some $\delta>0$ for the given partition $\left\{J_{k}, k=1, \cdots, N\right\}$, and that $m\left(\bigcup_{k=1}^{N-1} J_{k}\right) \geqq 1-\epsilon / 2$. 
Since $T^{N-k_{0-1}} f$ is constant on $J_{N-1}$ and zero on its complement, there exists $\delta_{1}>0$ such that $T^{N-k_{0}-1} f=\delta_{1} \psi_{J_{N-1}}$. Let $k_{1}$ be the least integer such that

$$
m\left(J_{k_{1}}\right)=\max _{1 \leqq k \leqq N-2} m\left(J_{k}\right),
$$

and let $\left\{A_{j}, j=1, \cdots, j_{0}\right\}$ be pairwise disjoint subsets of $J_{k_{1}}$ such that, with $\gamma=(1 / 2 B) \delta_{1} m\left(J_{N-1}\right)$,

$$
\text { (i) } \sum_{j=1}^{j_{1}} m\left(A_{j}\right)=\left(1-\frac{\epsilon}{2}\right) m\left(J_{k_{1}}\right)
$$

(ii) $\quad m\left(A_{j}\right) \leqq \frac{\gamma}{(j+1) N}, \quad 1 \leqq j \leqq j_{0}$.

That $\left\{A_{k}\right\}$ can be chosen to satisfy (2.1) follows easily since

$$
\sum_{j=1}^{\infty} \frac{\gamma}{(j+1) N}=+\infty \text {. }
$$

We define a partition $\left\{J_{k}^{1}, k=1, \cdots,\left(j_{0}+1\right)(N-1)+1\right\}$ as follows: Define

$$
\begin{aligned}
& B_{0}=c\left(\bigcup_{j=1}^{j_{0}} T^{-k_{1}+1} A_{j}\right) \cap J_{1}, \\
& B_{j}=T^{\left\llcorner k_{1}+1\right.} A_{j}, \quad j=1, \cdots, j_{0},
\end{aligned}
$$

and set

$$
\begin{array}{ll}
J_{k}^{1}=T^{k-1} B_{0}, & k=1, \cdots, N-1, \\
J_{k}^{1}=T^{k-N} B_{1}, & k=N, \cdots, 2 N-2, \\
J_{k}^{1}=T^{k-\left(j_{0}(N-1)+1\right)} B_{j_{0}}, & k=j_{0}(N-1)+1, \cdots,\left(j_{0}+1\right)(N-1), \\
J_{\left(j_{0}+1\right)(N-1)+1}^{1}=J_{N} . &
\end{array}
$$

It follows that the regular map $T^{\prime}$ of $\left\{J_{\mathbf{k}}^{1}\right\}$ is an extension of $T$, and that

$$
\frac{T^{\prime n}\left(\delta_{1} \psi_{J_{N-1}}\right)}{N-1+n} \geqq 2 B
$$

almost everywhere on $J_{N+n-1}^{1}, n=1, \cdots, j_{0}(N-1)$. Equation (2.2) implies that

$$
\sup _{K \leqq n \leqq\left(j_{0}+1\right)(N-1)}(1 / n) T^{n-1} f \geqq B
$$


almost everywhere on $C^{1}=\bigcup_{k=n}^{\left(y_{0}+1\right)(-1)} J_{k}^{1}$. The measure of $C^{1}$ is, by construction, $(1-\epsilon / 2)$ of the measure of $\bigcup_{k=1}^{N-1} J_{k}$.

In applying Lemma 2 to the invariant measure problem we may assume at the outset that $f>\delta$ and we may therefore obtain a further simplification by omitting the proof of (i), (ii) and (iii).

We state the theorem as follows:

TheOREM. If $a_{1}, a_{2}$, are positive constants, then there exists an invertible positive isometry $T$ of $L_{1}(0,1)$ and a positive function $f \in L_{1} \cap L_{\infty}$ such that $\|f\|_{1} \leqq a_{1},\|f\|_{\infty} \leqq a_{2}$ and such that

$$
\lim _{n \rightarrow \infty} \inf . \frac{1}{n} \sum_{k=0}^{n-1} T^{k} f=0
$$

and

$$
\underset{n \rightarrow \infty}{\lim . \sup } \frac{1}{n} \sum_{k=0}^{h-1} T^{k} f=\infty
$$

almost everywhere.

Proof. The theorem follows at once by successive applications of Lemmas 1 and 2 to any initial partition of the unit interval $(\epsilon \rightarrow 0)$ and to any function satisfying the norm conditions of the theorem.

\section{BIBLIOGRAPHY}

1. N. Dunford and J. Schwartz, Convergence almost everywhere of operator averages, J. Math. Mech. 5 (1956), 129-178.

2. R. Chacon, Convergence of operator averages, Ergodic theory, Academic Press, New York, 1963, pp. 89-120.

3. P. Halmos, Invariant measures, Ann. of Math. (2) 48 (1947), 735-754.

4. E. Hopf, The general temporally discrete Markov process, J. Math. Mech. 3 (1954), 13-45.

5. D. Ornstein, On invariant measures, Bull. Amer. Math. Soc. 66 (1960), 297-300.

Brown UNIVERSITY 